\title{
A mathematical description accounting for the superfluous hydrogen evolution and the inductive behaviour observed during electrochemical measurements on magnesium
}

\author{
M. Curioni ${ }^{\text {a, }}$, L. Salamone ${ }^{\text {a, b }}$, F. Scenini ${ }^{\text {a }}$, M. Santamaria ${ }^{\text {b }}$, M. Di Natale ${ }^{\text {c }}$ \\ ${ }^{a}$ School of Materials, Corrosion and Protection Centre, Material Performance Centre, University of Manchester, M139PP, Manchester, United Kingdom \\ ${ }^{\mathrm{b}}$ Electrochemical Materials Science Laboratory, DICAM, Università di Palermo, 90128, Palermo, Italy \\ ${ }^{\mathrm{c}}$ Department of Mathematics and Applications, Università degli Studi di Milano Bicocca, 20125, Milano, Italy
}

\section{A R T I C L E I N F O}

\section{Article history:}

Received 13 February 2018

Received in revised form

12 April 2018

Accepted 16 April 2018

Available online 17 April 2018

Keywords:

Magnesium

Inductive behaviour

Superfluous hydrogen evolution

Corrosion

\begin{abstract}
A B S T R A C T
When electrochemical techniques are used to probe the surface of corroding magnesium with the aim of obtaining quantitative information on the corrosion process, two peculiarities are generally observed: i) with anodic polarization, the rate of hydrogen evolution increases instead of decreasing and ii) during electrochemical impedance spectroscopy measurements, an inductive contribution is often observed at the low-frequency end of the spectra. The presence of these two phenomena clearly has an impact on the methodology that should be applied to correctly estimate corrosion rates from electrochemical data. The aim of this work is to provide a general mathematical description of the corroding magnesium surface that, under minimal a priori assumptions regarding the reaction kinetics, can account simultaneously for both superfluous hydrogen evolution and inductive response. The mathematical results are consistent with the suggestion that the superfluous hydrogen evolution is mainly related to the increase of the surface of the active corrosion front during anodic polarization. Further, the obtained results show that the inductive response is expected when, at the corrosion front, oxidation of magnesium proceeds faster than hydrogen evolution.
\end{abstract}

๑ 2019 The Authors. Published by Elsevier Ltd. This is an open access article under the CC BY license (http://creativecommons.org/licenses/by/4.0/).

\section{Introduction}

The electrochemical behaviour of magnesium surfaces is important for many applications since it determines the corrosion resistance of magnesium components, the efficiency of primary magnesium batteries [1-3] and of sacrificial anodes [4-7] for cathodic protection and the lifetime of resorbable implants in the human body [8-10]. In an aqueous environment, however, the electrochemical behaviour of magnesium is relatively complex. This is due to the fact that magnesium has an equilibrium potential for oxidation that is considerably lower than the potential for proton reduction resulting in hydrogen evolution over a wide $\mathrm{pH}$ range. Further, the mixed oxide-hydroxide film covering the magnesium surface is only partially protective in neutral or acidic $\mathrm{pH}$, so the corrosion resistance of magnesium is relatively poor [11].

Regarding the response of corroding magnesium surfaces to

\footnotetext{
* Corresponding author.

E-mail address: michele.curioni@manchester.ac.uk (M. Curioni).
}

electrochemical measurements, three peculiarities are important and specifically: i) the rate of hydrogen evolution ('superfluous hydrogen evolution' or 'negative difference effect') increases with increasing anodic polarization, for example during a potentiodynamic polarization experiment [11-21], ii) the cathodic activity of the magnesium surface ('cathodic activation') increases as a result of corrosion either at the free corrosion potential or following anodic polarization [22-26] and iii) an inductive behaviour generally is generally observed when performing electrochemical impedance spectroscopy measurements at the free corrosion potential [10,20,27-29].

Regarding the first effect, i.e. the superfluous hydrogen evolution, several interpretations have been proposed in the past, mainly related either to the formation of univalent magnesium $[13,30,31]$, or to the detachment of metallic chunks of magnesium followed by their oxidation away from the electrode [32]. Such theories have been largely dismissed, since recent works indicate that the increased rate of hydrogen evolution with increasing polarization is associated with the rupture of the partially protective film at the corrosion front that results in direct exposure of metallic 
magnesium to the solution. Due to the available large potential difference, additional hydrogen evolution takes place at the corrosion front [18-21,33-36]. The phenomenon is illustrated in Fig. 1, where a cross sectional view of a corroding magnesium electrode is presented, together with a schematic of the associated processes. The current that maintains active the corrosion front (at the centre of the image) is provided by hydrogen evolution on cathodically active regions. These regions consist of intermetallic particles located on the uncorroded part of the electrode, ahead of the corrosion front (left part of the image), or on the cathodically active regions located behind the corrosion front (right part of the images). At the corrosion front, the film is ruptured due to the simultaneous presence of an anodic current and of depassivating conditions, for example comparatively low $\mathrm{pH}$ and/or presence of chloride ions. As the film ruptures, the metal is directly exposed to the electrolyte (or covered by a chloride-rich film that offers virtually no protection to the underlying surface) and additional hydrogen is evolved. In this region, streams of very small bubbles are generated instead of comparatively large bubbles generated on the cathodic sites, because the dissolving surface is intrinsically unstable and doesn't allow the forming bubbles to anchor. This situation also produces an increase in the magnesium oxidation rate, since at the corrosion front an additional current associated to hydrogen evolution is produced and, consequently, an additional magnesium oxidation current is generated [18]. A very similar mechanism has been suggested to occur on aluminium, also displaying superfluous hydrogen evolution during anodic polarization in chloride-containing environments due to local rupture of the protective oxide film [36-38].

Fajardo and Frankel [34] have recently developed a kinetic model which accounts for these processes on magnesium. In particular, they have shown for exponential kinetics that, if the reaction coefficient associated with magnesium oxidation is larger than the one associated with hydrogen evolution on the active corrosion front, then the hydrogen evolution rate is expected to increase with increasing potential, due to the increasing area fraction associated with the corrosion front. In this work we follow broadly the general framework of Fajardo and Frankel, although some significant variations are introduced in order to account simultaneously of all the multiple overlapping processes.

In addition to the 'superfluous hydrogen evolution' an inductive response is generally observed when performing electrochemical
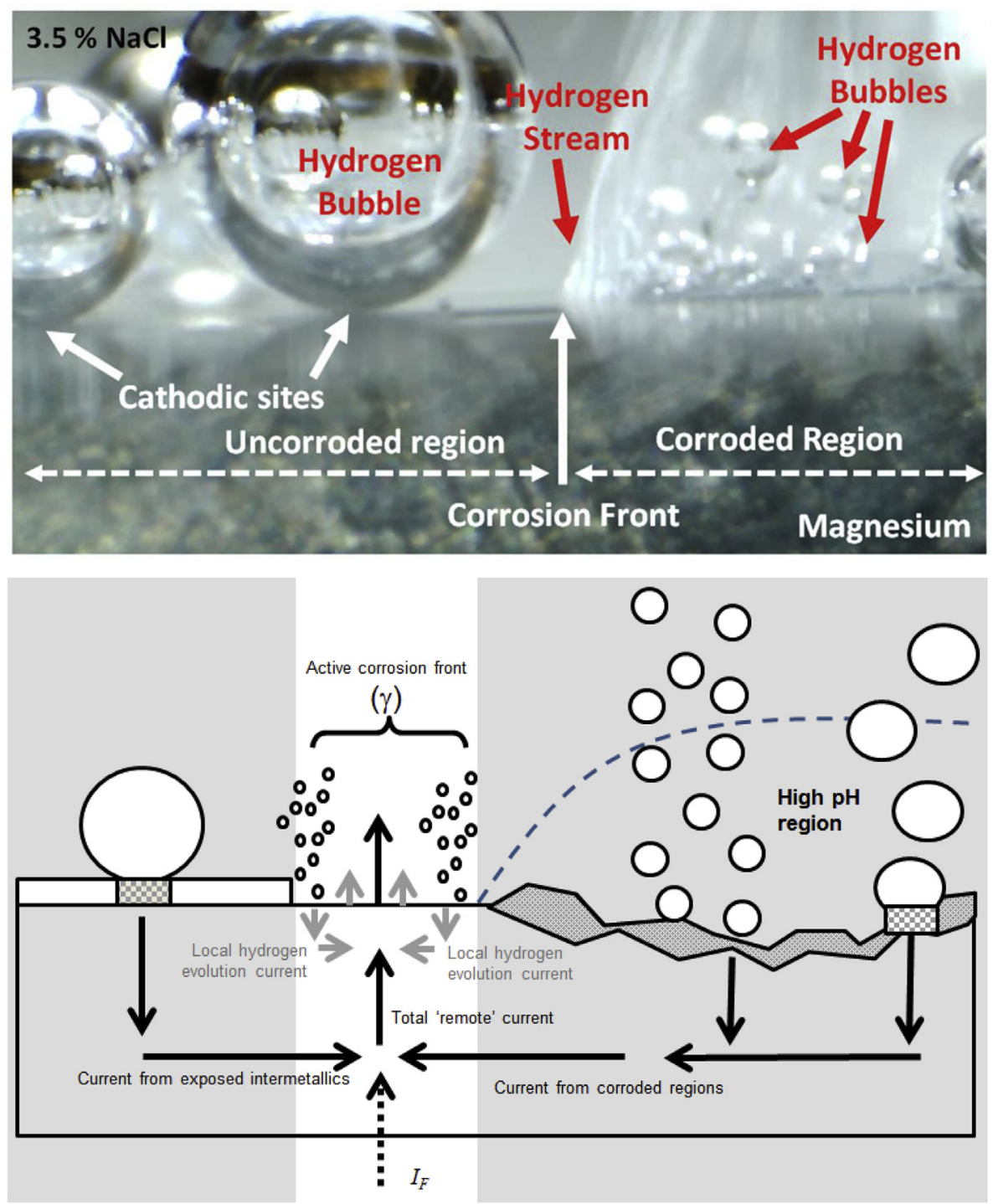

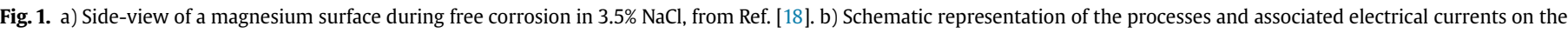
magnesium surface represented in Figure 1a, redrawn from Ref. [19]. 
impedance spectroscopy measurements on freely corroding magnesium [10,20,27-29]. Inductive responses in electrochemical processes are often associated with multi-step reactions, where one step produces an intermediate adsorbed layer that partially covers the surface so that subsequently the adsorbed species undergo a second reaction step [39]. In many kinetic models, the inductive response is associated with a variation in surface coverage of one of the intermediate species $[27,40,41]$. According to this interpretation, the inductive behaviour of corroding magnesium surfaces has been sometime related to the presence of an intermediate layer containing univalent magnesium ions [42,43]. Although this approach is formally correct and it provides an interpretation of the EIS response, recent efforts that aimed at experimentally revealing the presence of univalent magnesium have failed [44]. The absence of direct evidence and the improved understanding of the superfluous hydrogen evolution phenomena earlier discussed suggest that the observation of an inductive contribution during EIS measurements cannot be considered a supporting evidence for the formation of univalent magnesium ions. Further, it should be noted that postulating the presence of univalent magnesium to interpret both the superfluous hydrogen evolution and the inductive behaviour results in a paradox. If superfluous hydrogen evolution was due to formation of univalent magnesium and to subsequent reaction with water away from the electrode, then, even during EIS measurement, the electrochemical response should be the one associated with a single step reaction, i.e. the formation of univalent magnesium. In this case no inductive contribution should be observed. Vice versa, if univalent magnesium ions were adsorbed on the electrode, so generating an inductive contribution in the EIS response due to a two-steps reaction, then they would release the second electron into the metal. In this case, the overall process would not result in superfluous hydrogen evolution during anodic polarization. Thus, although postulating the existence of univalent magnesium could potentially account for superfluous hydrogen evolution and inductive response individually, the mechanisms till now proposed for each of the two individual processes are incompatible and cannot explain the both at the same time. Hence, electrochemical models should be introduced that could rigorously and simultaneously account both for superfluous hydrogen evolution during anodic polarization and for inductive contribution during EIS measurements.

The aim of this work is to provide a mathematical description, or model, for the corrosion processes on a magnesium surface that can account for the electrochemical response both under steady DC polarization and during EIS measurement. The model presented here doesn't require assumptions on the specific reaction kinetics for the underlying electrochemical processes. The individual reaction kinetics are treated as general functions of the electrode potential. As a result, the mathematical procedure is compact and relatively easy to follow and the theoretical conclusions that are drawn are independent of the specific underlying kinetics. In a future work, exponential kinetics for the individual reactions will be considered in detail.

\section{Electrochemical model}

\subsection{General framework}

The qualitative schematic of Fig. $1 \mathrm{~b}$ is redrawn in Fig. 2, such as the currents associated with each electrochemical process can be clearly and schematically defined. The model assumes that a fraction of the electrode surface is covered by a film, without differentiation between the air-formed film and the corrosion products film, and the remaining fraction of the electrode surface is occupied by the corrosion front, where the film is absent. At both locations,
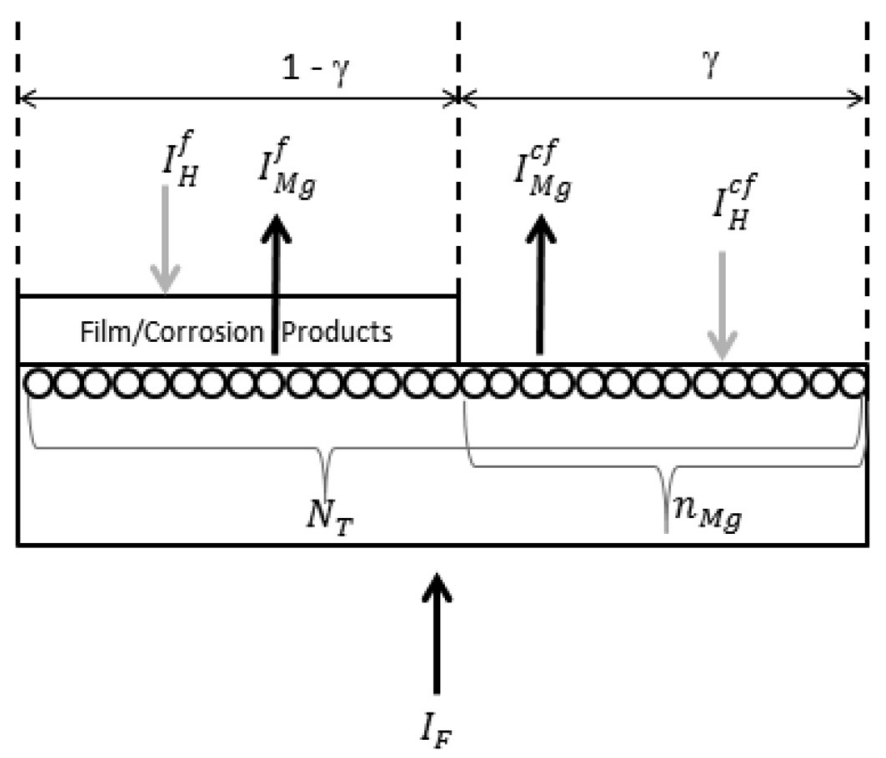

Fig. 2. Schematic representation of the individual currents associated with the processes of magnesium oxidation $\left(I_{M g}^{f}, I_{M g}^{c f}\right)$ and of hydrogen evolution $\left(I_{H}^{f}, I_{H}^{c f}\right)$ on completely non-filmed $\left(I_{M g}^{c f}, I_{H}^{c f}\right)$ or completely filmed $\left(I_{M g}^{f}, I_{H}^{f}\right)$ surfaces; $\gamma$ represents the area fraction of the electrode associated with the active corrosion front, where the magnesium atoms $\left(n_{M g}\right)$ don't support a film.

the filmed regions and the corrosion front, two reactions are always present: the (anodic) oxidation of magnesium atoms to form divalent magnesium cations

$M g \rightarrow M g^{2+}+2 e^{-}$

and the (cathodic) reduction of protons from water to form hydrogen gas

$2 \mathrm{H}^{+}+2 e^{-} \rightarrow \mathrm{H}_{2}$.

In the following discussion, the substrate supporting a specific reaction (corrosion front or filmed region) is indicated by a superscript (' $c f$ ' and ' $f$ ' respectively) whereas the reaction (hydrogen evolution or magnesium oxidation) is indicated by a subscript (' $H$ ' and ' $M g$ ' respectively).

Overall, four currents are simultaneously present on the corroding surface, specifically: i) $I_{M g}^{f}$, the current associated with the magnesium atoms that oxidize and subsequently form a film; ii) $I_{M g}^{c f}$, the current associated with the oxidation of magnesium atoms at the corrosion front, directly injected into the electrolyte and not forming a film; iii) $I_{H}^{f}$, the current associated with the hydrogen evolving from the filmed regions; iv) $I_{H}^{c f}$ the current associated with the hydrogen evolving from the active corrosion front.

Defining $\gamma$ as the area fraction of the electrode associated with the active corrosion front as schematically represented in Fig. 2, the individual currents can be expressed as

$I_{M g}^{f}=(1-\gamma) \dot{i}_{M g}^{f}$

$I_{M g}^{c f}=\gamma i_{M g}^{c f}$

$I_{H}^{f}=-(1-\gamma) i_{H}^{f}$ 
$I_{H}^{c f}=-\gamma i_{H}^{c f}$

where $i_{M g}^{f}, i_{M g}^{c f}, i_{H}^{f}, i_{H}^{c f}\left(\mathrm{~A} \mathrm{~m}^{-2}\right)$ are the potential-dependent magnitudes of the current densities associated with the reaction of magnesium oxidation $\left(i_{M g}^{f}, i_{M g}^{c f}\right)$ and of hydrogen evolution $\left(i_{H}^{f}, i_{H}^{c f}\right)$ on completely non-filmed $\left(i_{M g}^{c f}, i_{H}^{f f}\right)$ or completely filmed $\left(i_{M g}^{f}, i_{H}^{f}\right)$ surfaces. The currents associated with a reaction on a substrate, $I_{r}^{S}$, have units of current density. In other words, $i_{M g}^{f}, i_{M g}^{c f}, i_{H}^{f}, i_{H}^{c f}$ account for the fundamental reaction kinetics associated with the individual processes, while $I_{M g}^{f}, I_{M g}^{c f}, I_{H}^{f}, I_{H}^{c f}$ are the actual macroscopic current densities generated on the electrode surface as a result of each individual process occurring on a fraction of the electrode area.

Regardless of the detailed reaction kinetics, which are not discussed in this paper, $i_{M g}^{f}, i_{M g}^{c f}$ are the potential dependent functions associated with anodic reactions and consequently they are positive, they approach zero for large cathodic polarization and they increase in magnitude with increasing anodic polarization. On the other hand, $i_{H}^{f}, i_{H}^{c f}$ are the potential dependent functions associated with cathodic currents and consequently they are positive (the negative sign for cathodic currents is added when calculating $I_{H}^{f}$ and $I_{H}^{c f}$ ), they approach zero for large anodic polarization and their magnitude increases for increasing cathodic polarization. Based on the previous considerations, it is also reasonable to assume that the derivatives of $i_{M g}^{f}$ and $i_{M g}^{c f}$ with respect to the potential are positive and the derivatives of $i_{H}^{f}$ and $i_{H}^{c f}$ are negative, for any value of electrode potential.

Based on Eqs. (3)-(6), the faradaic current, which can be measured experimentally and is the sum of all the anodic and cathodic currents, is

$I_{F}=(1-\gamma)\left(i_{M g}^{f}-i_{H}^{f}\right)+\gamma\left(i_{M g}^{c f}-i_{H}^{c f}\right)$

The total anodic current, associated with magnesium oxidation

$I_{M g}=(1-\gamma) i_{M g}^{f}+\gamma i_{M g}^{c f}$

And the total cathodic current, associated with hydrogen evolution is

$I_{H}=-(1-\gamma) i_{H}^{f}-\gamma i_{H}^{c f}$

The corrosion front can be thought as being formed by a number of 'active' sites, i.e. non filmed regions, similarly to what proposed previously by Fajardo and Frankel in their kinetic model [34]. The active sites form when the oxide/hydroxide layer covering the metal surface is locally damaged, due to the combined presence of an anodic current and depassivating conditions, such as chloride ions and/or low $\mathrm{pH}$. Once formed, an active site can de-activate, for example due to the formation of a stable film, separating the metal from the electrolyte. It seems reasonable to assume that the time variation of the number of magnesium atoms forming active sites at the corrosion front, $n_{M g}\left(\mathrm{~mol} \mathrm{~m}^{-2}\right)$, is given by the difference between their formation rate, $r_{M g}\left(\mathrm{~mol} \mathrm{~m}^{-2} \mathrm{~s}^{-1}\right)$, and a 'deactivation' term, $n_{M g} \lambda$. Such deactivation term depends on the total number of active sites and on their average lifetime. The lifetime of an active site is likely to be affected by the composition of the environment. More aggressive environments, i.e. with low $\mathrm{pH}$ or with high chloride content, are likely to increase the lifetime of active sites, since stable film formation is difficult. Thus, the deactivation term can be written as the total number of active sites $n_{M g}$ times their frequency of deactivation $\lambda\left(\mathrm{s}^{-1}\right)$, where the frequency of deactivation $\lambda$ is inversely proportional to the lifetime of the active sites [10]. Using this approach, we can write:

$\frac{d n_{M g}}{d t}=r_{M g}-n_{M g} \lambda$.

Defining the fraction of the surface coverage of the active sites as

$\gamma=\frac{n_{M g}}{N_{T}}$

where $\left.N_{T}(\mathrm{~mol} \mathrm{~m})^{-2}\right)$ is the total number of atoms forming the electrode surface, it is possible to calculate the time variation of the fraction of the surface coverage $\gamma$ :

$\frac{d \gamma}{d t}=\frac{r_{M g}}{N_{T}}-\lambda \gamma$

It appears reasonable to assume that the rate of formation of the active sites is related to the current density associated with magnesium oxidation on the filmed regions. If the current density passing across the film in the presence of chloride increases, film rupture becomes more likely and new active corrosion sites, not supporting a stable film, are formed. As a result, the surface coverage of the active corrosion sites increases and the total anodic current redistributes among the filmed regions and the preexisting and newly formed active corrosion sites. This assumption is different from the one which is at the basis of the model of Fajardo and Frankel [34] and which considers the rate of formation of active sites to be proportional to the overall current associated with magnesium oxidation. Their assumption has the drawback that the surface coverage of the active sites is not restricted to the value of one for large anodic polarization.

Regardless, considering acceptable that the rate of formation of the active sites is related to the current density on the filmed regions and assuming in first approximation that it is directly proportional to the current density, then the rate of formation of atoms generating active sites can be written as

$r_{M g}=\rho \frac{I_{M g}^{f}}{z F}$

where: $\rho$ is a constant (dimensionless) between zero and one, that accounts for the fraction of the current passing across a filmed region that results in the formation of a defect in the film, and hence of an active corrosion site, $z$ is the equivalent number ( 2 for magnesium) and $F$ is the Faraday's constant.

\subsection{Steady state}

At the steady state, the surface coverage of the active corrosion front is constant, hence

$\frac{d \gamma}{d t}=0$

As a consequence, the expression of $\bar{\gamma}$, where the straight accent indicates quantities associated with steady-state conditions, can be readily obtained from equations (12)-(14)

$\bar{\gamma}=\frac{\rho \bar{i}_{M g}^{f}}{\rho i_{M g}^{f}+z F N_{T} \lambda}$ 
Then the derivative of the surface coverage with respect to the electrode potential is

$\frac{d \bar{\gamma}}{d E}=\frac{\rho z F N_{T} \lambda \frac{d \bar{i}_{M g}^{-f}}{d E}}{\left(\rho i_{M g}+z F N_{T} \lambda\right)^{2}}$

It is immediately evident that $\bar{\gamma}$ approaches zero when the current associated with the magnesium atoms forming a film approaches zero, and approaches the value of one for large anodic currents associated with magnesium forming a film, regardless of the underlying kinetics. This qualitative behaviour appears to be physically acceptable. The derivative of $\bar{\gamma}$ with respect to the potential is always positive.

The charge transfer resistances Rassociated with each individual reaction (Eqs. (3)-(6)) are equal to the reciprocal of the derivative of the individual current $I$ associated with that reaction with respect to the electrode potential, maintaining all other quantities constant, and calculated in $\bar{E}$ [39]. Specifically:

$\frac{1}{R_{M g}^{f}}=\frac{d \hat{i}_{M g}^{f}}{d E}(1-\bar{\gamma})$

$\frac{1}{R_{M g}^{c f}}=\frac{d \bar{i}_{M g}^{c f}}{d E} \bar{\gamma}$

$\frac{1}{R_{H}^{f}}=-\frac{d \bar{i}_{H}^{f}}{d E}(1-\bar{\gamma})$

$\frac{1}{R_{H}^{c f}}=-\frac{d \bar{i}_{H}^{c f}}{d E} \bar{\gamma}$

The faradaic current and the total magnesium and hydrogen currents (Eqs. (7)-(9)) are expressed as

$$
\begin{aligned}
\bar{I}_{F}= & \left(1-\frac{\rho \bar{i}_{M g}^{f}}{\rho j \hat{i}_{M g}^{f}+z F N_{T} \lambda}\right)\left(\overline{i_{M g}^{f}}-\overline{i_{H}^{f}}\right)+\left(\frac{\rho \bar{i}_{M g}^{f}}{\rho \overline{i_{M g}^{f}}+z F N_{T} \lambda}\right)\left(\bar{i} \bar{i}_{M g}\right. \\
& \left.-\bar{i}_{H}^{c f}\right)
\end{aligned}
$$

$\bar{I}_{M g}=\left(1-\frac{\rho \bar{i}_{M g}^{f}}{\rho \bar{i}_{M g}^{f}+z F N_{T} \lambda}\right) \bar{i}_{M g}^{f}+\left(\frac{\rho \bar{i}_{M g}^{f}}{\rho i_{M g}^{f}+z F N_{T} \lambda}\right) \bar{i} c f$

$\bar{I}_{H}=-\left(1-\frac{\rho \hat{i}_{M g}^{f}}{\rho \bar{i} \hat{i}_{M g}+z F N_{T} \lambda}\right) \bar{i}_{H}^{f}-\left(\frac{\rho \hat{i}_{M g}^{f}}{\rho \bar{i} \hat{i}_{M g}^{f}+z F N_{T} \lambda}\right) \bar{i}_{H}^{c f}$

\subsection{Response to a small sinusoidal potential perturbation}

The linear response to a small sinusoidal potential perturbation, such as that applied during an electrochemical impedance measurement, can be evaluated reconsidering Eqs. (12) and (13). In particular, it is possible to write $\frac{d \gamma}{d t}=\frac{\rho}{z F N_{T}} I_{M g}^{f}-\lambda \gamma$

Following Orazem and Tribollet [39], the oscillating component of $I_{M g}^{f}$ can be expressed as

$\tilde{I}_{M g}^{f}=\left.\frac{\partial I_{M g}^{f}}{\partial E}\right|_{\bar{E}} \tilde{E}+\left.\frac{\partial I_{M g}^{f}}{\partial \gamma}\right|_{\bar{\gamma}} \tilde{\gamma}=\frac{1}{R_{M g}^{f}} \tilde{E}-\tilde{i}_{M g}^{f} \tilde{\gamma}$

According to eqs. (24) and (25), the oscillating component of the coverage fraction, $\tilde{\gamma}$, can be expressed as

$\tilde{\gamma} j \omega=\frac{\rho}{z F N_{T}}\left(\frac{1}{R_{M g}^{f}} \tilde{E}-\tilde{i}_{M g}^{f} \tilde{\gamma}\right)-\lambda \tilde{\gamma}$

giving

$\tilde{\gamma}=\frac{\frac{\rho}{R_{M g}^{f}}}{z F N_{T}(j w+\lambda)+\rho \hat{i}_{M g}^{f}} \tilde{E}$.

The faradaic impedance $Z_{F}$ is defined by

$Z_{F}=\frac{\tilde{E}}{\tilde{I}_{F}}$

and the reciprocal of the faradaic impedance is

$\frac{1}{Z_{F}}=\frac{\tilde{I}_{F}}{\tilde{E}}$

where $\tilde{I}_{F}$ is the oscillating component of the faradaic current. $\tilde{I}_{F}$ can be expressed as [39].

$\tilde{I}_{F}=\left.\frac{\partial I_{F}}{\partial E}\right|_{\bar{E}} \tilde{E}+\left.\frac{\partial I_{F}}{\partial \gamma}\right|_{\bar{\gamma}} \tilde{\gamma}$

where

$\left.\frac{\partial I_{F}}{\partial E}\right|_{\bar{E}}=\frac{1}{R_{M g}^{f}}+\frac{1}{R_{M g}^{c f}}+\frac{1}{R_{H}^{f}}+\frac{1}{R_{H}^{c f}}$

$\left.\frac{\partial I_{F}}{\partial \gamma}\right|_{\bar{\gamma}}=\bar{i}_{H}^{f}+\bar{i}_{M g}^{c f}-\bar{i}_{H}^{c f}-\bar{i}_{M g}^{f}$

and all $R_{M g}^{f}, R_{M g}^{c f}, R_{H}^{f}, R_{H}^{c f}$ and $i_{H}^{f}, i_{M g}^{c f}, i_{H}^{c f}, i_{M g}^{f}$ are calculated for the value of $\bar{E}$. As a result, the oscillating component of the faradaic current can be expressed as

$$
\begin{aligned}
\tilde{I}_{F}= & \left(\frac{1}{R_{M g}^{f}}+\frac{1}{R_{M g}^{c f}}+\frac{1}{R_{H}^{f}}+\frac{1}{R_{H}^{c f}}\right) \tilde{E}+\left(\tilde{i}_{H}^{f}+\bar{i}_{M g}^{c f}-\bar{i}_{H}^{c f}\right. \\
& \left.-\bar{i}_{M g}^{f}\right) \frac{\frac{\rho}{R_{M g}^{f}}}{z F N_{T}(j w+\lambda)+\rho \bar{i}_{M g}^{f}} \tilde{E}
\end{aligned}
$$

Combining eq. (29) and eq. (33), the expression of the reciprocal of the faradaic impedance can be readily obtained: 


$$
\begin{aligned}
\frac{1}{Z_{F}}= & \left(\frac{1}{R_{M g}^{f}}+\frac{1}{R_{M g}^{c f}}+\frac{1}{R_{H}^{f}}+\frac{1}{R_{H}^{c f}}\right)+\left(\bar{i}_{H}^{f}+\bar{i}_{M g}^{c f}-\bar{i}_{H}^{c f}\right. \\
& \left.-\bar{i}_{M g}^{f}\right) \frac{\frac{\rho}{R_{M g}^{f}}}{z F N_{T}(j w+\lambda)+\rho \bar{i}_{M g}^{f}}
\end{aligned}
$$

\subsection{Intrinsic limitations of the presented model}

In the interest of clarity, and to limit the complexity, the model presented here has an intrinsic limitation related to the way the currents on the film have been defined. All currents have been defined as the product of a term which accounts for the kinetic of an individual reaction on a specific substrate, for example $i_{M g}^{f}$, and a term which accounts for the surface fraction of the electrode where the reaction occurs, for example $(1-\gamma)$. Thus the overall current associated, for example, to the oxidation of magnesium resulting in film formation has been defined as $I_{M g}^{f}=(1-\gamma) i_{M g}^{f}$. In principle, $i_{M g}^{f}$, and the other similar kinetics, should not be a function of time. However, during the initial corrosion transient, when the magnesium electrode is partially silvery and partially black, the part of the filmed electrode corresponding to $(1-\gamma)$ actually comprises some silvery areas, supporting a relatively protective air formed film, and some areas supporting a comparatively less protective corrosion product film, as evident from the time lapse images of a corroding magnesium surface reported in Fig. 3. It is likely that the current associated with magnesium oxidation resulting in film formation follows different kinetics on the silvery and dark regions due to the less protective nature of the corrosion product film on the dark regions compared to the air-formed film on the silvery regions. Further, it is well known that hydrogen evolution proceeds faster on the dark regions due to cathodic activation after corrosion [22-26]. These aspects, however, are not explicitly considered in this model, so in strict terms, the model presented here is valid only for an electrode where the extension of the silvery regions is negligible compared to that of the dark regions or, vice versa, for an electrode where the extension of the dark regions is negligible compared to that of the silvery regions. In practice, the change in ratio between silvery and dark regions over time might produce an apparent time dependence of the kinetic terms $i_{M g}^{f}$ and $i_{H}^{f}$ during the transient when the electrode progressively becomes dark.

\section{Discussion}

\subsection{Superfluous hydrogen evolution}

The possibility that the corroding magnesium surface generates the so called 'negative difference effect', or superfluous hydrogen evolution generation can be evaluated by considering eq. (9) at the steady state and rearranging such as

$H_{2}=\frac{1}{2 F}\left[(1-\bar{\gamma}) \bar{i}_{H}^{f}+(\bar{\gamma}) \bar{i}_{H}^{c f}\right]$

where $\mathrm{H}_{2}$ is the rate of hydrogen evolution with units of $\mathrm{mol} \mathrm{cm} \mathrm{cm}^{-2}$ $\mathrm{s}^{-1}$. Then,

$\frac{d H_{2}}{d E}=\frac{1}{2 F}\left[\frac{d \bar{\gamma}}{d E}\left(\bar{i}_{H}^{c f}-\bar{i}_{H}^{f}\right)+(1-\bar{\gamma}) \frac{d \bar{i}_{H}^{f}}{d E}+\bar{\gamma} \frac{d i_{H}^{c f}}{d E}\right]$

It is evident that superfluous hydrogen evolution is possible for polarization above the corrosion potential if and only if $\frac{d H_{2}}{d E}>0$, that is

$\frac{d \bar{\gamma}}{d E}\left(\bar{i}_{H}^{c f}-\bar{i}_{H}^{f}\right)+(1-\bar{\gamma}) \frac{d \hat{i}_{H}^{f}}{d E}+\bar{\gamma} \frac{d \bar{i}_{H}^{c f}}{d E}>0$

or equivalently

$\frac{d \bar{\gamma}}{d E}\left(\bar{i}_{H}^{c f}-\bar{i}_{H}^{f}\right)>(1-\bar{\gamma})\left(-\frac{d f_{H}^{f}}{d E}\right)+\bar{\gamma}\left(-\frac{d \bar{i}_{H}^{c f}}{d E}\right)$.

Note that in inequality 38 both sides are always positive, since at any potential $\bar{i}_{H}^{c f}>\bar{i}_{H}^{f}>0,0<\gamma<1, \frac{d i_{H}^{f}}{d E}<0, \frac{d i_{H}^{c f}}{d E}<0, \frac{d \bar{\gamma}}{d E}>0$. Then, the following further inequalities can be obtained

$$
\begin{aligned}
& \frac{d \bar{\gamma}}{d E} \bar{i}_{H}^{c f}>\frac{d \bar{\gamma}}{d E}\left(\bar{i}_{H}^{c f}-\bar{i}_{H}^{f}\right) \quad \text { and } \quad(1-\bar{\gamma})\left(-\frac{d \bar{i}}{d E}\right)+\bar{\gamma}( \\
& \left.-\frac{d \bar{i}_{H}^{c f}}{d E}\right)>\bar{\gamma}\left(-\frac{d \bar{i}_{H}^{c f}}{d E}\right) .
\end{aligned}
$$

which can be inserted into inequality 38 to give

$$
\frac{d \bar{\gamma}}{d E} \bar{i}_{H}^{c f}>\bar{\gamma}\left(-\frac{d \bar{i}_{H}^{c f}}{d E}\right)
$$

In order to point out the intensity $\tilde{i}_{M g}$, it is convenient to write 40 as

$$
\frac{d \bar{\gamma}}{\bar{\gamma}}>\frac{\left(-\frac{d \bar{i}_{H}^{-f}}{d E}\right)}{\bar{i}_{H}^{c f}}
$$

and to calculate the ratio $\frac{\frac{d \bar{\gamma}}{\bar{\gamma}}}{\bar{\gamma}}$. From 15 to 16 , it follows immediately

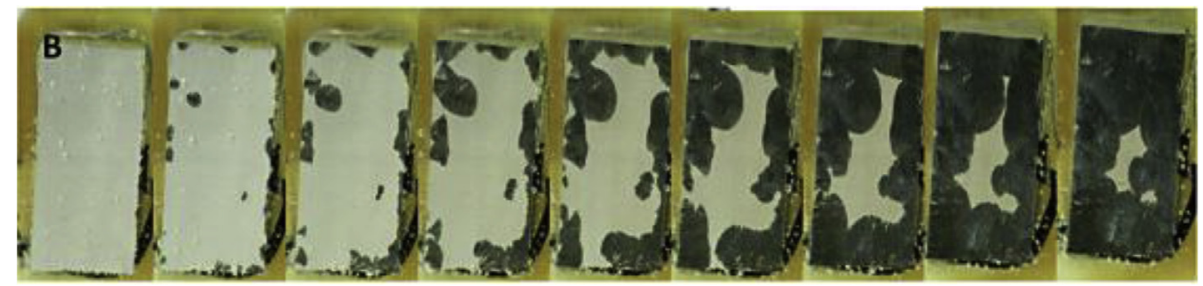

Fig. 3. Time evolution of the appearance of a freely corroding magnesium surface in $3.5 \% \mathrm{NaCl}$, from Ref. [21]. 
$\frac{\frac{d \bar{\gamma}}{d E}}{\bar{\gamma}}=\frac{z F N_{T} \lambda}{\rho i_{M g}^{f}+z F N_{T} \lambda} \frac{\frac{d i_{M g}^{-f}}{d E}}{\overline{i_{M g}^{f}}}$

And, since $\frac{z F N_{T} \lambda}{\rho i_{M g}^{f}+z F N_{T} \lambda}<1, \bar{i}_{M g}^{f}>0, \frac{d i_{M g}^{-f}}{d E}>0$, then

$\frac{d \bar{\gamma}}{\bar{\gamma} E}<\frac{\frac{d i_{M g}^{f}}{d E}}{i_{M g}^{f}}$

Now, from inequalities 41 and 43 it is possible to obtain that

$\frac{\frac{d \bar{i}_{M g}}{d E}}{\bar{i}_{M g}^{f}}>\frac{\left(-\frac{d i_{H}^{-c f}}{d E}\right)}{\bar{i}_{H}^{c f}}$

which represents a condition that $\bar{i}_{M g}^{f}, \bar{i}_{H}^{c f}$ and their derivatives have to satisfy in order that the 'negative difference effect' or superfluous hydrogen evolution can occur.

It should be noted that 44 can also be written as

$\frac{d}{d E}\left(\ln \bar{i}_{M g}^{f}\right)>-\frac{d}{d E}\left(\ln \bar{i}_{H}^{c f}\right)$

Inequalities 41-45 indicate that the phenomenon of superfluous hydrogen evolution is related to the increase in area of the active corrosion front during anodic polarization. When such increase in area during anodic polarization dominates over the decrease of the hydrogen current due to the reaction kinetic, then superfluous hydrogen evolution is possible. This becomes more evident considering the example of exponential kinetics; in this case inequality 45 reduces to the comparison between the Tafel coefficient for the reaction of magnesium oxidation on filmed regions and the Tafel coefficient for the reaction of hydrogen evolution on non-filmed regions. In other words, hydrogen evolution is possible if the Tafel coefficient for the hydrogen evolution on the filmed region is higher than the Tafel coefficient for hydrogen evolution on the non-filmed region. Qualitatively, the result is similar, but more general, than that obtained by Fajardo and Frankel [10].

\subsection{Physical meaning of the equivalent circuit elements for EIS fitting}

Generally, electrochemical impedance spectra are interpreted by using an equivalent circuit approach, where the values associated with each equivalent circuit element are used to extract information on the processes occurring on the electrode surface. Thus, it is important to explicitly obtain the relationships among the quantities introduced in the fundamental model and the values of the equivalent circuit elements. In compact form, equation (34) can be expressed as:

$\frac{1}{Z_{F}}=\frac{1}{R_{T}}+\frac{A}{j \omega+B}$

where

$\frac{1}{R_{T}}=\frac{1}{R_{M g}^{f}}+\frac{1}{R_{M g}^{c f}}+\frac{1}{R_{H}^{f}}+\frac{1}{R_{H}^{c f}}$

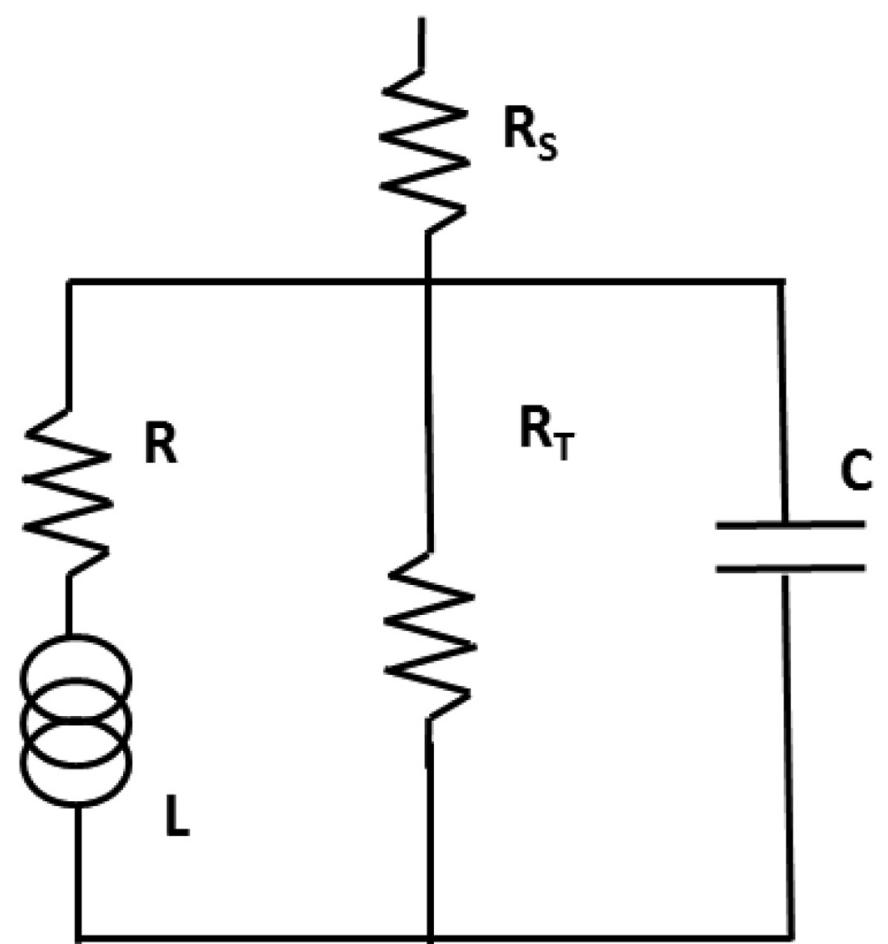

Fig. 4. Equivalent circuit used to fit the experimental data of Fig. 5.

$$
\begin{aligned}
& A=\frac{\rho\left(\bar{i}_{H}^{f}+\bar{i}_{M g}^{c f}-\bar{i}_{H}^{c f}-\bar{i}_{M g}^{f}\right)}{z F N_{T} R_{M g}^{f}} \\
& B=\lambda+\frac{\rho}{z F N_{T}} \bar{i}_{M g}^{f}
\end{aligned}
$$

It is immediately evident that when A takes positive values, then $Z_{F}$ shows an inductive behaviour [39]. If the equivalent circuit of Fig. 4 is used to fit the experimental EIS data (where a capacitor C has been added to account for both film capacitance and double layer capacitance, and a resistor $\mathrm{R}_{\mathrm{S}}$ has been added to account for the solution resistance), it is possible to relate the values of inductance and resistances in the equivalent circuit with the previous quantities, specifically:

$$
\begin{gathered}
L=\frac{1}{A}=\frac{z F N_{T} R_{M g}^{f}}{\rho\left(\bar{i}_{H}^{f}+\bar{i}_{M g}^{c f}-\bar{i}_{H}^{c f}-\bar{i}_{M g}^{f}\right)} \\
R=\frac{B}{A}=\frac{\left(z F N_{T} \lambda+\rho \bar{i}_{M g}^{f}\right) R_{M g}^{f}}{\rho\left(\bar{i}_{H}^{f}+\bar{i}_{M g}^{c f}-\bar{i}_{H}^{c f}-\bar{i}_{M g}^{f}\right)}
\end{gathered}
$$

And

$R / L=B=\lambda+\frac{\rho}{z F N_{T}} \bar{i}_{M g}^{f}$

Inductive behaviour during EIS measurement.

As mentioned previously, the presence of an inductive behaviour during an EIS measurement is associated with the positive sign of the term $\mathrm{A}$ in equation (46). It is immediately evident that the sign of $A$ is determined by the individual reaction kinetics only, and it is positive if and only if 
$\bar{i}_{M g}^{c f}-\bar{i}_{M g}^{f}>\bar{i}_{H}^{c f}-\bar{i}_{H}^{f}$

Remembering that $\bar{i}_{H}^{f}, \bar{i}_{M g}^{c f}, \bar{i}_{H}^{c f}, \bar{i}_{M g}^{f}$ account for the fundamental reaction kinetics and don't account for the actual area where the individual reaction takes place, it appears reasonable to assume that, at the corrosion potential, the fundamental kinetics for one reaction on filmed regions are much slower than kinetics for the same reaction on non-filmed regions, and therefore

$\bar{i}_{M g}^{c f} \gg \bar{i}_{M g}^{f}$

And

$\bar{i}_{H}^{c f} \gg \bar{i}_{H}^{f}$

In this case, inequality 53 can be approximated by

$\bar{i}_{M g}^{c f}>\bar{i}_{H}^{c f}$

Thus, if the kinetic of magnesium dissolution on non-filmed regions is faster than the kinetic of hydrogen evolution on the same regions, an inductive behaviour is observed. It is immediately evident that this condition is verified for free corrosion of magnesium since the corrosion front, supporting both magnesium dissolution, $i_{M g}^{c f}$, and hydrogen evolution, $\bar{i}_{H}^{c f}$, is a net anode compared to the filmed regions. This implies that, at the free corrosion potential, $\bar{i}_{M g}^{c f}>\bar{i}_{H}^{c f}$. It should also be noted that, with anodic polarization, $\bar{i}_{M g}^{c f}$ increases while $\bar{i}_{H}^{c f}$ decreases. As a consequence, inductive behaviour is expected to be more common during anodic polarization than during cathodic polarization, which is qualitatively consistent with experimental findings.

\subsection{Discussion of experimental data}

Fig. 6 presents the fitting results of the experimental data of Fig. 5 by using the equivalent circuit of Fig. 4 , where $R_{T}, L$ and $R$ correspond to the terms in equations (47), (50) and (51) respectively, a capacitance $C$ has been added to account for charge separation at the double layer and across the filmed regions, and a resistance $R_{S}$ has been added to account for the solution resistance. The data presented in Fig. 5 are redrawn from Ref. [20], where full experimental details can be found. In brief, the spectra were acquired sequentially in two solutions with different concentrations of sodium chloride, namely $3.5 \%$ and $0.35 \%$ wt., at natural $\mathrm{pH}$.

It is evident (Fig. 6) that the total resistance decreases as a function of time. Since the total resistance is given by the parallel between the individual reaction resistances, the overall behaviour is likely to be dominated by the lower individual resistance. As corrosion proceeds, cathodic activation occurs; thus the value of the resistance associated with the hydrogen evolution on the filmed regions will decrease, and more current will be available to sustain the corrosion front. As a result, the surface coverage of the corrosion front will increase, producing a reduction of the
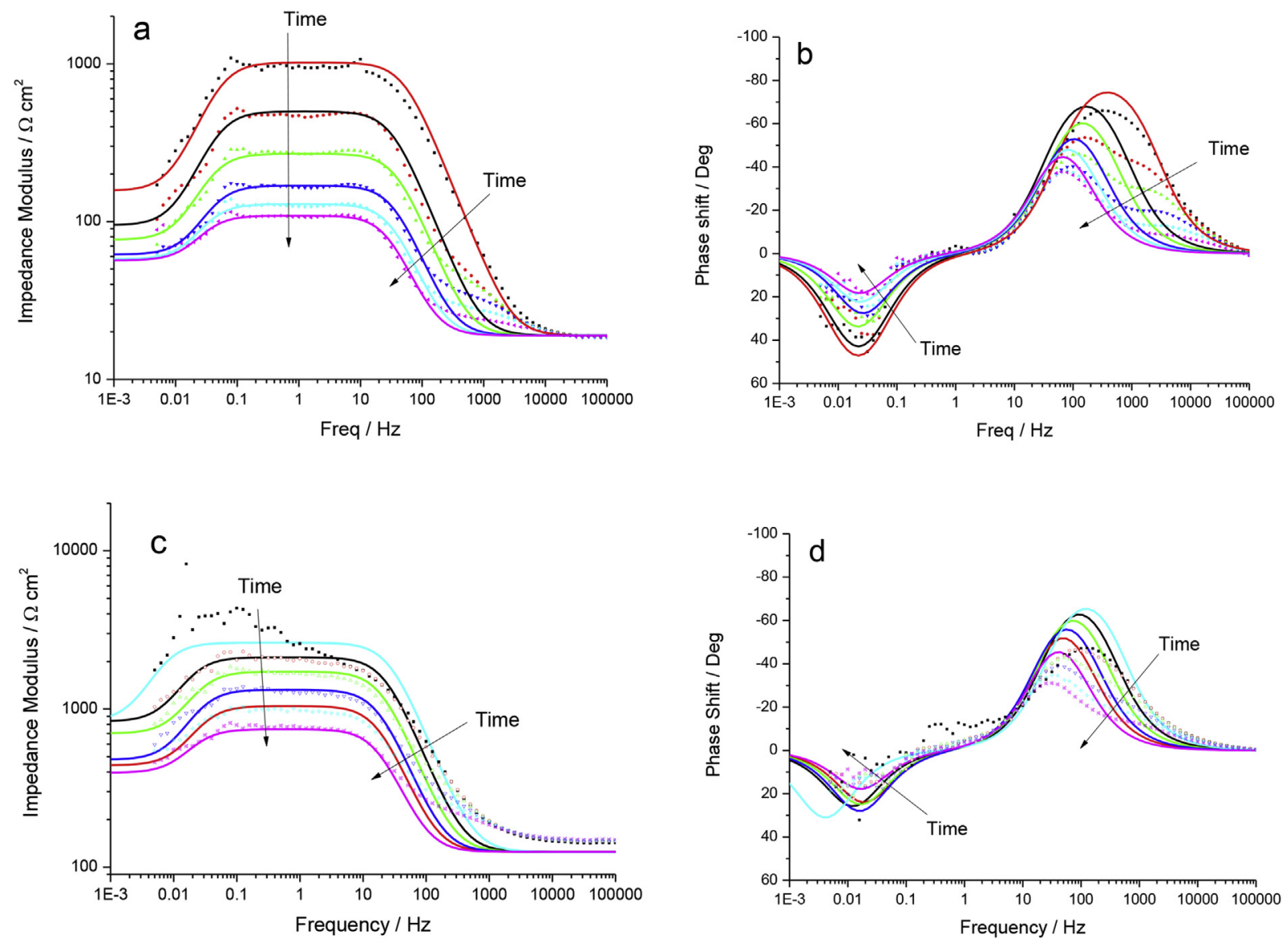

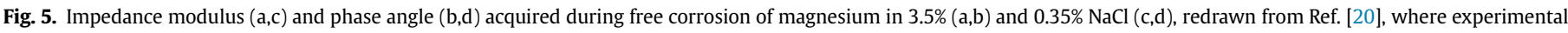
details are given. Symbols represent experimental values, lines are simulated spectra obtained with the equivalent circuit of Fig. 4. 

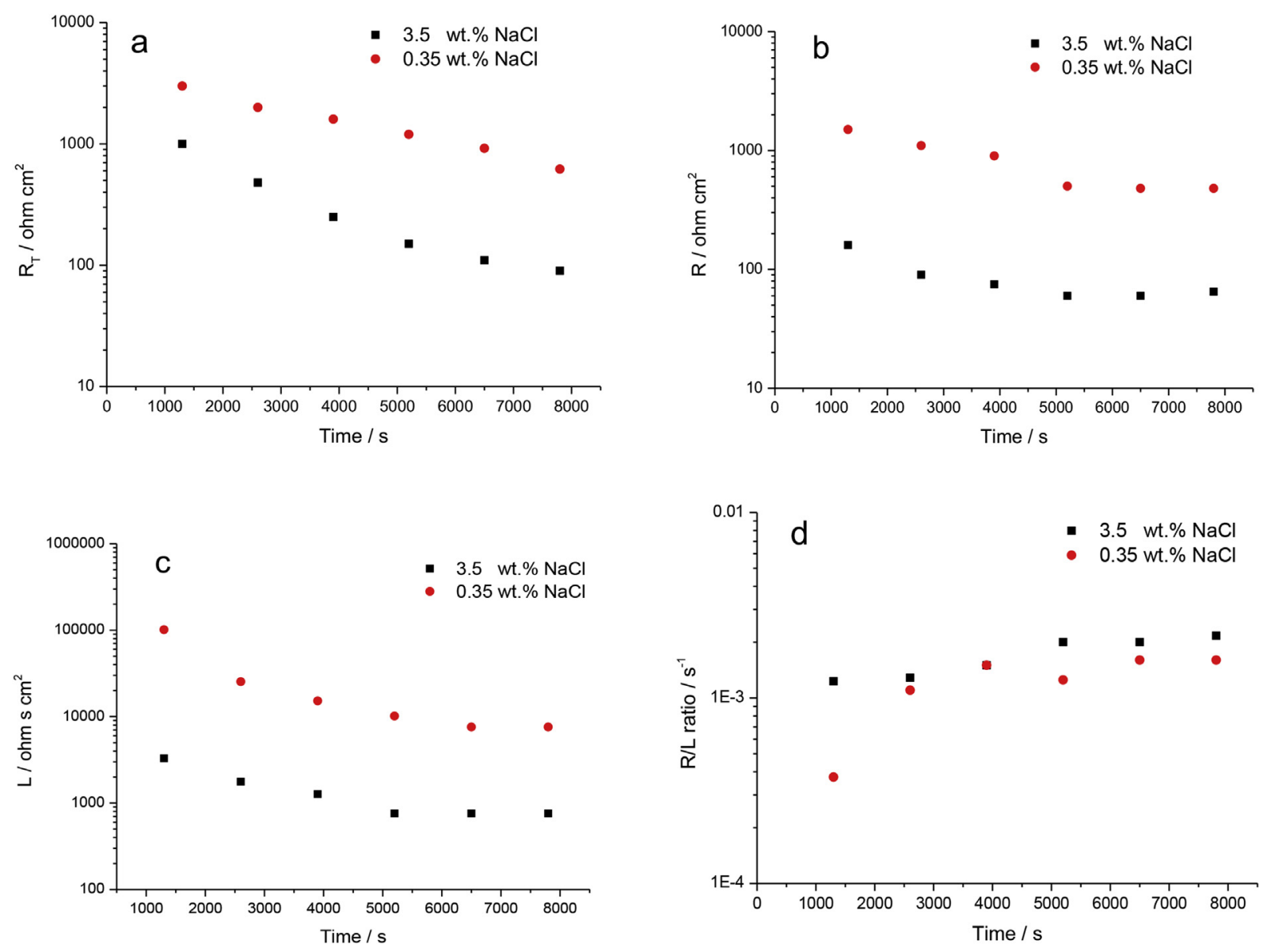

Fig. 6. Time evolution of the electrical parameters obtained by fitting the spectra of Fig. 5 by using the equivalent circuit of Fig. 4 ; $R_{T}$ (a), $R(\mathrm{~b}$ ), $L$ (c) and of the ratio $R / L$ (d).

resistances associated with the oxidation of magnesium at the corrosion front and of hydrogen evolution at the corrosion front. The resistance associated with magnesium oxidation on the filmed regions might also decrease due to the less protective nature of the corrosion product film compared to the air formed film. Although the general trend with respect to the time is similar, the total resistance is higher for the magnesium corroding in the less concentrated chloride solution. Here, $R_{M g}^{f}$ is likely to be higher, due to the more stable film. Thus, overall, the experimentally observed decrease in $R_{t}$ with time and with chloride concentration agrees well with the model presented here.

The value of $\mathrm{R}$ and $\mathrm{L}$ appear to decrease with time, but significant differences are observed in different concentrations. However, considering the complex expressions of $\mathrm{R}$ and $\mathrm{L}$ it is not possible to draw a final conclusion without making additional assumptions on the kinetics of the individual processes, since many terms appear simultaneously both at the numerator and at the denominator. The discussion of specific reaction kinetics will be the focus of a future work.

However, it is interesting to observe the behaviour of the ratio between the resistance $\mathrm{R}$ and the inductance $\mathrm{L}$. This number only depends on the values of $\rho, \lambda$ and $i_{M g}^{f}$. The parameter $\lambda$ has the dimensions of $\mathrm{s}^{-1}$ and it has been introduced to account for the deactivation of the active corrosion sites. The most likely process that induces the de-activation of an active corrosion site is the formation of a partially protective film covering the active site. Thus, it is likely that the main parameter affecting the value of $\lambda$ is the composition of the electrolyte. High $\mathrm{pH}$ and low chloride amounts would result in high value of $\lambda$, since they promote the rapid formation of a relatively stable film, and therefore the newlyformed corrosion sites will rapidly deactivate.

On the other hand, $\rho$ (dimensionless) was defined as the fraction of the current flowing across filmed regions that form a new active corrosion site. Similarly to $\lambda$, also $\rho$ is likely to depend on electrolyte composition. At high $\mathrm{pH}$ and low chloride content, the film is relatively stable and only a very small proportion of the current forming the film will induce the formation of an active corrosion site; consequently $\rho$ will have low value. Vice versa, at low $\mathrm{pH}$ and high chloride content, the film is less stable and therefore a larger proportion of the current forming the film will generate a new active corrosion site. In this case the value of $\rho$ will be higher and closer to 1 .

The value of $B$ appears to increase slightly with time. All the terms composing the expression of $B$ are expected to be constant with time, except for the term $\hat{i}_{M g}^{f}$ that might increase slightly, due to the propagation of the corroded areas (supporting a less perfect film) at the expenses of the uncorroded areas (supporting a more protective air formed film). Thus, the time increase of $B$ can be attributed to the propagation of the dark areas on the specimen surface.

\section{Conclusions}

A general mathematical description for the electrochemical response of corroding magnesium surfaces has been developed in this work. Four individual current contributions are considered: i) 
the current associated with oxidation of magnesium followed by film formation, ii) the current associated with oxidation of magnesium at the corrosion front, iii) the current associated with hydrogen evolution on the filmed regions and iv) the current associated with hydrogen evolution at the corrosion front. In order to develop the mathematical model, it is not required to assume a priori any specific kinetics for the individual reactions; consequently the results apply to a wide range of experimental conditions. Nevertheless, the results allows rationalizing both the formation of superfluous hydrogen evolution during anodic polarization and the presence of inductive behaviour during EIS measurement. In particular, the model is compatible with the idea that the phenomenon of superfluous hydrogen evolution is related to the increase in area of the active corrosion front during anodic polarization. If such increase in area during anodic polarization dominates on the decrease of the hydrogen current due to the reaction kinetic, then superfluous hydrogen evolution is possible. Similarly, the results indicate that the inductive response observed during EIS measurements can be a direct consequence of the fact that, at the corrosion front, the local current associated with magnesium oxidation exceeds the local current associated with hydrogen evolution.

\section{Data access statement}

All data supporting this study are provided in graphical form in the manuscript.

\section{Acknowledgments}

EPSRC is acknowledged for provision of financial support through the Lightform Grant (EP/R001715/1). L.S. gratefully acknowledges the financial support provided by the University of Palermo. Dr Simone Marzani - Prof Mark Orazem are acknowledged for discussion - correspondence.

\section{References}

[1] D. Aurbach, Y. Gofer, Z. Lu, A. Schechter, O. Chusid, H. Gizbar, Y. Cohen, V. Ashkenazi, M. Moshkovich, R. Turgeman, E. Levi, J. Power Sources. 97-98 (2001) 28-32.

[2] D. Aurbach, Z. Lu, A. Schechter, Y. Gofer, H. Gizbar, R. Turgeman, Y. Cohen, M. Moshkovich, E. Levi, Nature 407 (2000) 724-727.

[3] D. Aurbach, G.S. Suresh, E. Levi, A. Mitelman, O. Mizrahi, O. Chusid, M. Brunelli, Adv. Mater. 19 (2007) 4260-4267.

[4] M. Andrei, F. Di Gabriele, P.L. Bonora, D. Scantlebury, Mater. Corros. 54 (2003) $5-11$.

[5] F. Guadarrama-Muñoz, J. Mendoza-Flores, R. Duran-Romero, J. Genesca,
Electrochim. Acta 51 (2006) 1820-1830

[6] J.G. Kim, S.J. Koo, Corrosion 56 (2000) 380-388.

[7] G.T. Parthiban, T. Parthiban, R. Ravi, V. Saraswathy, N. Palaniswamy, V. Sivan, Corrosion Sci. 50 (2008) 3329-3335.

[8] M.P. Staiger, A.M. Pietak, J. Huadmai, G. Dias, Biomaterials 27 (2006) $1728-1734$

[9] F. Witte, Acta Biomater. 6 (2010) 1680-1692.

[10] Y. Liu, M. Curioni, Z. Liu, Electrochim. Acta 264 (2018) 101-108.

11] G.L. Song, A. Atrens, Adv. Eng. Mater. 1 (1999) 11-33.

[12] G. Song, A. Atrens, Adv. Eng. Mater. 5 (2003) 837-858.

[13] G. Song, A. Atrens, D. St John, X. Wu, J. Nairn, Corrosion Sci. 39 (1997) 1981-2004.

[14] G. Song, A. Atrens, D. Stjohn, J. Nairn, Y. Li, Corrosion Sci. 39 (1997) 855-875.

[15] G.S. Frankel, A. Samaniego, N. Birbilis, Corrosion Sci. 70 (2013) 104-111.

[16] G. Williams, N. Birbilis, H.N. McMurray, Electrochem. Commun. 36 (2013) $1-5$.

[17] S. Thomas, N.V. Medhekar, G.S. Frankel, N. Birbilis, Curr. Opin. Solid State Mat. Sci., (0000).

[18] Y. Yang, F. Scenini, M. Curioni, Electrochim. Acta 198 (2016) 174-184.

[19] M. Curioni, J. Torrescano-Alvarez, Y. Yang, F. Scenini, Corrosion 73 (2017) $463-470$.

[20] M. Curioni, F. Scenini, T. Monetta, F. Bellucci, Electrochim. Acta 166 (2015 $372-384$

[21] M. Curioni, Electrochim. Acta 120 (2014) 284-292.

[22] R. Liu, S. Thomas, J. Scully, G. Williams, N. Birbilis, Corrosion (2016).

23] R.L. Liu, M.F. Hurley, A. Kvryan, G. Williams, J.R. Scully, N. Birbilis, Sci. Rep. 6 (2016).

[24] R.L. Liu, J.R. Scully, G. Williams, N. Birbilis, Electrochim. Acta 260 (2018) 184-195.

[25] R.L. Liu, S. Thomas, J.R. Scully, G. Williams, N. Birbilis, Corrosion 73 (2017) 494-505.

[26] J.A. Yuwono, N. Birbilis, R. Liu, Q. Ou, Q. Bao, N.V. Medhekar, J. Electrochem. Soc. 164 (2017) C918-C929.

[27] V. Shkirskiy, A.D. King, O. Gharbi, P. Volovitch, J.R. Scully, K. Ogle, N. Birbilis, ChemPhysChem 16 (2015) 536-539.

[28] G. Baril, G. Galicia, C. Deslouis, N. Pébère, B. Tribollet, V. Vivier, J. Electrochem. Soc. 154 (2007) C108-C113.

[29] A.D. King, N. Birbilis, J.R. Scully, Electrochim. Acta 121 (2014) 394-406.

[30] R.L. Petty, A.W. Davidson, J. Kleinberg, J. Am. Chem. Soc. 76 (1954) 363-366.

[31] T.R. Thomaz, C.R. Weber, T. Pelegrini, L.F.P. Dick, G. Knörnschild, Corrosion Sci 52 (2010) 2235-2243.

[32] G.A. Marsh, E. Schaschl, J. Electrochem. Soc. 107 (1960) 960-965.

[33] S. Fajardo, G.S. Frankel, Electrochim. Acta 165 (2015) 255-267.

34] S. Fajardo, G.S. Frankel, Electrochem. Commun. 84 (2017) 36-39.

[35] S. Fajardo, C.F. Glover, G. Williams, G.S. Frankel, Electrochim. Acta 212 (2016) 510-521.

[36] G.S. Frankel, S. Fajardo, B.M. Lynch, Faraday Discuss 180 (2015) 11-33.

[37] M. Curioni, F. Scenini, Electrochim. Acta 180 (2015) 712-721.

[38] C. Laurent, F. Scenini, T. Monetta, F. Bellucci, M. Curioni, Npj Mater. Degrad. 1 (2017) 6.

[39] M.E. Orazem, B. Tribollet, Electrochemical Impedance Spectroscopy, 2008.

[40] I. Epelboin, M. Keddam, J. Electrochem. Soc. 117 (1970) 1052-1056.

[41] L. Péter, J. Arai, H. Akahoshi, J. Electroanal. Chem. 482 (2000) 125-138.

[42] J. Kubásek, D. Dvorský, M. Čavojský, D. Vojtěch, N. Beronská, M. Fousová J. Mater. Sci. Technol. 33 (2017) 652-660.

[43] G. Galicia, N. Pébère, B. Tribollet, V. Vivier, Corrosion Sci. 51 (2009) 1789-1794.

[44] A. Samaniego, B.L. Hurley, G.S. Frankel, J. Electroanal. Chem. 737 (2015) $123-128$. 